\title{
Anchoring of Ulex Europaeus Agglutinin to Chitosan Nanoparticles-in- Microparticles and Their in Vitro Binding Activity to Bovine Submaxillary Gland Mucin
}

\author{
Feng-Qian Li, ${ }^{*}, a$ Yi-Bo FeI, ${ }^{b}$ Xu Chen, ${ }^{a}$ Xian-Ju Qin,,${ }^{a}$ Ji-Yong Liu,,${ }^{b}$ Quan-Gang Zhu, ${ }^{b}$ and \\ Jin-Hong $\mathrm{Hu}^{*, b}$ \\ ${ }^{a}$ Shanghai Eighth People's Hospital; $8^{\#}$ CaoBao Road, Shanghai 200235, China: and ${ }^{b}$ Changhai Hospital, Second \\ Military Medical University; $168^{\#}$ ChangHai Road, Shanghai 200433, China. \\ Received February 8, 2009; accepted July 6, 2009; published online July 9, 2009
}

\begin{abstract}
Focused on the natural biodegradable material of chitosan (CS), this investigation concerned its spray-dried nanoparticles-in-microparticles (NiMPs) modified with ulex europaeus agglutinin (UEA). Chitosan nanoparticles were obtained by ionotropic gelation process with pentasodium tripolyphosphate as gelatinizer. Then UEA lectin was bound onto the CS nanoparticles activated by glutaraldehyde. The conjugated spherical UEA-CS-NiMPs, prepared by spray drying method, exhibited $12-85 \%$ coupling efficiency of UEA depending upon the amount of activator glutaraldehyde. And the UEA-grafted particles showed additional higher binding tendency with bovine submaxillary gland mucin as compared to the plain chitosan microparticles. Furthermore, the activity and intrinsic fucose-specificity of UEA were still maintained after the covalent modification. It is thus evident that the UEA anchored CS-NiMPs might be used as a potential drug delivery system targeted to the specific regions of gastrointestinal tract.
\end{abstract}

Key words chitosan; nanoparticle-in-microparticle; ulex europaeus agglutinin; fucose; bovine submaxillary gland mucin

The tremendous progress witnessed in the field of biotechnology with respect to discovery of therapeutic and antigenic proteins has propelled the need for development of suitable delivery systems for these and other macromolecules. Among the various routes of drug delivery, the oral route is mostly attractive due to the ease of administration and very high patient compliance. Unfortunately, oral vaccination is largely ineffective due to substantial degradation of antigens by gastric acid and proteolytic enzymes. And various strategies for delivery systems have been devised for effective oral vaccine delivery. Microparticles/nanoparticles delivery systems are particularly useful to protect the antigen in the gastrointestinal (GI) tract and hold potential to enhance the efficacy of oral vaccine. ${ }^{1)} \mathrm{M}$ cells targeted oral delivery systems hold promise to improve the efficacy of the oral vaccines. It seems likely that vaccine efficacy could be enhanced by anchoring $\mathrm{M}$ cells specific ligands (e.g., lectins) on to the bioactive carriers. ${ }^{2,3)}$

Lectins, comprised a structurally very diverse class of proteins or glycoproteins of nonimmunological origin, was found in organisms ranging from viruses and plants to humans, characterized by their ability to bind sugar molecules with considerable specificity. And therefore, they are capable of specific recognition of and reversible binding to carbohydrate determinants of complex glycoconjugates, without altering the covalent structure of the recognized glycosyl ligands. ${ }^{4-6)}$ Lectin receptors are expressed on various cells such as endothelial cells, hepatocytes, macrophages, monocytes and lymphocytes. They are efficient in recognizing the complex oligosaccharide epitopes, which are also present on the cell surface or could be exogenous glycoconjugate ligands mimics of endogenous carbohydrate epitopes. ${ }^{7)}$ This property suggests that they play an important role in biological-recognition events. In recent years, lectins, described as "second generation mucoadhesives," have been proposed as tools to offer some advantages either in specific drug delivery appli-

* To whom correspondence should be addressed. e-mail: fqlijr@gmail.com cations, to increase the residence time of the pharmaceutical form or to resist proteolytic degradation. ${ }^{8,9)}$

There is an interest in the grafting of lectins to the surface of biodegradable polymeric particles. This conjugation to polymeric nanoparticles would enhance their interactions with mucus and lectins, which have been shown to be efficient for promoting particle translocation. ${ }^{10-13)}$ Additionally, lectins could probably be useful for targeting specific areas of the GI tract, due to the heterogenous distributions of sugars moieties along the normal or diseased intestine. ${ }^{14)} \mathrm{Up}$ to now, a lot of polymer nanoparticles have been conjugated with the selected lectin. $3,11,12,15,16)$

Chitosan (CS), mostly obtained from exoskeletons of marine arthropods, is the second abundant polysaccharide and a cationic polyelectrolyte present in nature. The characteristics of fine biocompatibility, biodegradability, non-toxicity and the ability to open epithelial tight junctions have rendered it to be widely applicable in the pharmaceutical fields. ${ }^{17)} \mathrm{Re}$ cently, a novel nanoparticles-in-microsphere oral system (NiMOS) has been developed using a "double emulsion-like" technique and evaluated as potential drug and gene delivery in specific regions of the GI tract for therapeutic and vaccination purposes. ${ }^{18,19)}$ The advantages of NiMOS might involve in the achieved high drug entrapment efficiency, controlled drug release and potential enhanced absorption. However, few reports refer to the preparation of chitosan based NiMOS. According to our knowledge, little information was available for the coupling of lectins to chitosan or its micro/nanoparticles.

In this paper, CS nanoparticles (CS-NPs) were prepared by ionotropic gelation process with pentasodium tripolyphosphate (TPP) as the cross-linking agent. After activation of the amino groups in CS by glutaraldehyde, ulex europaeus agglutinin (UEA) was anchored to the CS-NPs. Then the colloidal solution of UEA coupled CS-NPs was spray dried to obtain the lectin modified microparticulate system (UEA- 
CS-NPs-MPS). The bio-binding activity investigation of the prepared UEA-CS-NPs-MPS was carried out by bovine submaxillary gland mucin (BSM) assay. Additionally, its sugarspecificity to fucose was also examined in the present work.

\section{Experimental}

Materials Chitosan (CS) with the deacetylation degree above $95 \%$ and the molecular weight $(\mathrm{Mw})$ of $40-80 \mathrm{kDa}$ was supplied by RuJi Bio-pharmaceutical Co. (Shanghai, China). Glutaraldehyde and pentasodium tripolyphosphate (TPP) were obtained from the Chemical Agent Station, Medicine Group of China. Ulex europaeus agglutinin (UEA), bovine submaxillary gland mucin (BSM) and $\alpha$-(L)-fucose were purchased from Sigma-Aldrich Chemie GmbH (Steinheim, Germany). All other chemicals were of reagent grade and used as received.

Preparation of Chitosan Nanoparticles The chitosan nanoparticles were prepared according to the ionotropic gelation process as previously described. ${ }^{20,21)}$ Chitosan solution $(0.2 \%, \mathrm{w} / \mathrm{v})$ was obtained by dissolving chitosan in dilute acetic acid $(0.3 \%, \mathrm{v} / \mathrm{v})$ at room temperature and filtered through a $0.22 \mu \mathrm{m}$ filter to remove insolubles. Then, pentasodium tripolyphosphate (TPP) was directly dissolved in distilled water at $2 \mathrm{mg} / \mathrm{ml}(\mathrm{w} / \mathrm{v})$. Thereafter, the ionic gelification of chitosan in the aqueous medium was achieved by addition of TPP solution corresponding to a $0.2 \%$ CS solution $(w / v)$ stirred $(600 \mathrm{rpm})$ at room temperature. And the weight ratio of chitosan to TPP was kept at $6: 1(\mathrm{w} / \mathrm{w})$ for the construction of chitosan nanoparticles. The achieved CS-NPs were collected by centrifugation at $20000 \mathrm{rpm}$ for $30 \mathrm{~min}$, and the supernatants were discarded.

Preparation of UEA Anchored Chitosan Nanoparticles UEA were covalently conjugated to the surface of the CS-NPs by using glutaraldehyde as activator. A two step procedure, including the first activation step and then a coupling step was adopted. ${ }^{16}$

Sample of CS-NPs was activated as following. The obtained nanoparticles, centrifuged from $5 \mathrm{ml}$ of the above CS-NPs colloidal suspension, were dispersed in $500 \mu \mathrm{l}$ of phosphate-buffered saline (PBS, pH 7.4). Then, microcontent of glutaraldehyde $(0.25 \%, \mathrm{v} / \mathrm{v})$ was added into the colloida nanoparticles. The mixture was shaken gently for $6 \mathrm{~h}$ to activate the amino groups of CS. Thereafter, the activated CS-NPs were conjugated to the lectin. Briefly, the above suspension was centrifuged to remove the un-reacted glutaraldehyde and further washed three times in PBS (pH 7.4) to remove any remaining traces of glutaraldehyde which might otherwise crosslink the lectin molecules. Subsequently, $50 \mu$ l UEA contained PBS $(0.28 \%$, $\mathrm{w} / \mathrm{v}$ ) was added and the linkage process was made by incubation overnight at room temperature. As a result, conjugates were centrifuged $(20000 \mathrm{rpm}$, $20 \mathrm{~min}$ ) to remove the free lectin. And the UEA anchored CS nanoparticles (UEA-CS-NPs) were finally re-suspended in PBS (containing 3\% glycerol $(\mathrm{w} / \mathrm{v})$ and $0.2 \%$ sodium azide $(\mathrm{w} / \mathrm{v})$ as preservative) and stored at $4{ }^{\circ} \mathrm{C}$ until use.

Determination of the Amount of UEA Bound to CS-NPs In order to evaluate the lectin bound efficiency, the quantification method for UEA was established by gel permeability HPLC. The chromatographic system was equipped with two LC-10AT pumps (Shimadzu), a Rheodyne 7125 manual injection valve with $20 \mu \mathrm{l}$ sample loop, an SPD-10A UV detector (Shimadzu), and a G2000SWXL TSK-GEL column $(7.8 \mathrm{~mm} \times 300 \mathrm{~mm}, 5 \mu \mathrm{m}$, $\mathrm{TOSOH}$, Japan) used at room temperature. The mobile phase was $0.1 \mathrm{M}$ phosphate buffer $(\mathrm{pH} 7.0)$ operated at a flow rate of $0.5 \mathrm{ml} / \mathrm{min}$. And the wavelength was set at $280 \mathrm{~nm}$. After chromatographic analysis, areas of UEA were calculated, and the least square linear regression was used to fit the peak areas versus UEA concentration. After the removal of free lectin by centrifugations, $20 \mu \mathrm{l}$ of the clear supernatant was injected to determine the amount of unbound UEA.

The quantity of lectin attached to CS-NPs was calculated as the difference between the lectin initially added and the lectin which was recovered in solution after centrifugation. The UEA binding capacity (BC) to CS-NPs was calculated as indicated below:

$$
\mathrm{BC}=\left(W_{\text {total UEA }}-W_{\text {free UEA }}\right) / W_{\text {total UEA }} \times 100 \%
$$

$W_{\text {tatUEA }}$, total amount of UEA; $W_{\text {free UEA }}$, amount of free UEA not bound to CS-NPs.

Formulation of UEA-CS-NPs-MPS from UEA-CS-NPs by Spray Drying Considered the stability aspect and advantages of NiMOS, the aqueous colloidal suspension of UEA-CS-NPs was then microencapsulated into powdered NiMOS by spray drying method. The UEA-CS-NPs contained colloidal sample was spray dried through the two-flowed pressure nozzle $\left(0.7 \mathrm{~mm}\right.$, diameter) of the mini spray dryer (Büchi ${ }^{\mathbb{B}} \mathrm{B} 290$, Switzerland). The experimentally optimized process conditions to obtain UEA-CS-NPs-MPS were the following: inlet air temperature $120 \pm 1{ }^{\circ} \mathrm{C}$, outlet air temperature $60 \pm 1{ }^{\circ} \mathrm{C}$, aspirator percent $85 \%$, pump feed percent $22 \%$, Q flow $45 \pm 3$. The small droplets were dispersed into the hot air stream, leaving the aqueous solvent evaporate very quickly and the solid microparticles settled into the bottom collector. Finally, the obtained product of UEA-CS-NPs-MPS was harvested and kept under vacuum for $48 \mathrm{~h}$.

Confirmation of the Particle Morphology The morphology and surface appearance of UEA-CS-NPs-MPS powders were examined by scanning electron microscopy (SEM, XL-30E, Philips). The microparticle sample was coated with gold and observed at an accelerating voltage of $20 \mathrm{kV}$ with SEM

The Bound of UEA-CS-NPs-MPS to BSM in Vitro UEA-CS-NPsMPS was re-dispersed in PBS ( $\mathrm{pH}$ 7.4). And the investigation about the binding process of UEA-CS-NPs-MPS to BSM was carried out by mixing BSM contained PBS $(1.0 \mathrm{mg} / \mathrm{ml})$ with $20 \mathrm{mg}$ lectin-conjugated microparticle suspended in PBS $(15 \mu \mathrm{g}$ lectin $/ \mathrm{ml})$ for $3 \mathrm{~h}$. After incubation, the samples were centrifuged for $10 \mathrm{~min}$ at $10000 \mathrm{rpm}$, and aliquots of the supernatants were taken and assayed by the described gel permeability HPLC. The amount of interacted or bound mucin was calculated as the difference between the mucin added and the BSM remained in the supernatants of the samples. The plain microparticles without lectin, was also incubated with the same amount of mucin as the reference sample.

Competitive Inhibition Studies with Fucose For evaluation of the sugar-specificity of UEA-CS-NPs-MPS, competitive inhibition effect of fucose on BSM bound to lectin was studied. One milliliter of $\alpha$-(L)-fucose $(1 \mathrm{mg} / \mathrm{ml})$ aqueous solution was added to the BSM contained PBS and mixed them thoroughly. The binding properties about UEA-CS-NPs-MPS and the plain microparticles without lectin, were performed as described above.

\section{Results and Discussion}

The Formation of CS-NPs and UEA-CS-NPs Solubility of CS in water could be improved by the addition of an acid medium as a result of the protonation of the amino groups which therefore is loaded with positive charges. As the dropwise addition of TPP, the particles formation become possible based on the electrostatic attraction between the amino groups of CS and the negative groups of TPP. ${ }^{22)}$ Meanwhile the incorporation of ion TPP to CS solution, the opalescence indicated the formation process of nanoparticles with a size range of $200-500 \mathrm{~nm}$, a mean diameter of approximately $300 \mathrm{~nm}$ and positive charge $(12 \mathrm{mV}$, analyzed with a NicompTM 380 ZLS coulter, laser droppler velocimetry). The amino groups in the chitosan molecule might assign the polymer with the same reducibility as the proteins. ${ }^{23}$ In view of this property, the free chitosan was determined according to the MicroBCA Kit method. And the yield of CSNPs was evaluated to be about $80 \%$.

The association of lectins to particulate systems could be achieved by covalent linkage or adsorption process. Due to more stable than simple adsorption, a covalent linkage method is often used to bind the ligands to particles with the remained activity and specificity. The two-stage carbodiimide method was early used to covalently bind lectin to polymer nanoparticles. ${ }^{24)}$ Carbodiimide involves the activation of carboxylic acid groups to give $\mathrm{NH}$-activated groupings which could react with free amino groups of the ligand polypeptide chains. ${ }^{25}$ ) And the moiety should be coupled on the surface of the substrate before the chemical reaction between the two species taking place.

Presented with numerous of amino groups, chitosan is an excellent candidate for the preparation of conjugates, formed by the attachment of specific lectin molecules to the surface of CS nanoparticles. Glutaraldehyde might be very efficient in cross-linking CS as the high content of adjacent hydroxyl 


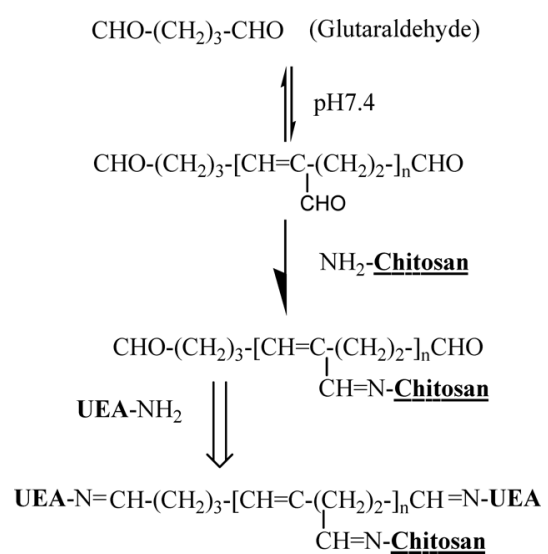

Fig. 1. Chemical Reactions Involved during the Activation of CS with Glutaraldehyde and Bound of UEA onto the Activated CS

groups in neighboring chains. In the present study, UEA was fixed by the two-stage glutaraldehyde coupling technique. Figure 1 described the reactional mechanism involved in this process. Polymerized glutaraldehyde molecules, resulted at neutral $\mathrm{pH}$ from an aldolic condensation, could react with the amino groups of CS and form an imine bond which is stabilized by the presence of a conjugated ethylenic bond. ${ }^{26)}$ Free aldehydic groups allow either the propagation of the crosslinking reaction or the fixation of exogenous molecules of UEA (Fig. 1). The structural formula of UEA-CS in Fig. 1 has not been decided yet. And the chemical structure of UEA-CS would be decided by NMR or FT-IR analysis in the further research work.

Effect of Glutaraldehyde Content on the UEA Binding Capacity to CS-NPs To better understand the quantitative integrity of the bound lectin, UEA was analyzed by gel permeability HPLC based upon the aqueous mobile phase system. As shown in Fig. 2, surface content of UEA on the activated CS-NPs was increased progressively depending on the amount of coupling agent. The binding yield of UEA in the grafting procedure was comprised between 12 and $85 \%$ depending mostly on the addition of $0.25 \%$ glutaraldehyde solution (v/v) (Fig. 2).

The necessary activation of CS-NPs before covalent coupling of the lectin might explain this trend. More lectin could react with the aldehyde groups covalently, as the rising content of glutaraldehyde added. While the cross-linking density of glutaraldehyde to CS increased, more effective sites which would react with lectins were available. So enhanced binding rates expected, and $85.3 \%$ UEA could be bound to CS-NPs activated with $200 \mu \mathrm{l}$ glutaraldehyde (Fig. 2). When the amount of glutaraldehyde rose to some extent above $200 \mu 1$, the binding capacity of UEA reached a steady platform. This phenomenon is perhaps because of the limitation or saturation of the binding sites on the activated CS-NPs.

Activity Verification of the Bound Lectin SEM of the spray-dried UEA-CS-NPs-MPS is shown in Fig. 3. The prepared microparticles, with a regular spherical shape and a particle size $<5 \mu \mathrm{m}$, were encapsulated from several CSNPs. Resulted from the dispersity of the sprayed fog droplets, the size distributions of the spray-dried NiMOS particles could be divided into two sections of $180-500 \mathrm{~nm}$ and $2-5 \mu \mathrm{m}$ (Fig. 3). The small solid particles with the

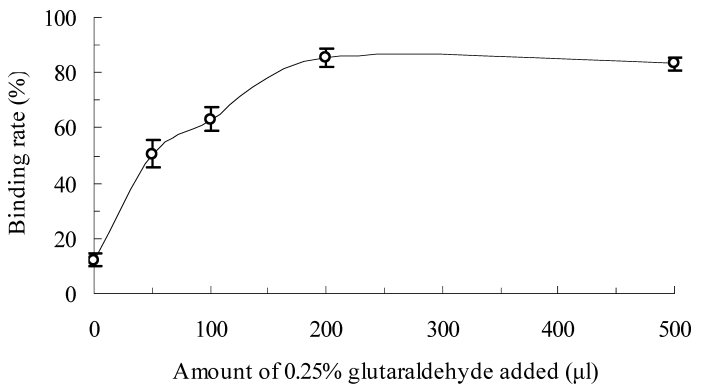

Fig. 2. The Effect of Different Amount of Glutaraldehyde on the Coupling Capacity of $50 \mu \mathrm{l}$ UEA Lectin $(2.8 \mathrm{mg} / \mathrm{ml}$ PBS $)$ to CS Nanoparticles Colloidal Solution ( $9 \mathrm{mg}$, Measured by MicroBCA Method) $(n=3)$

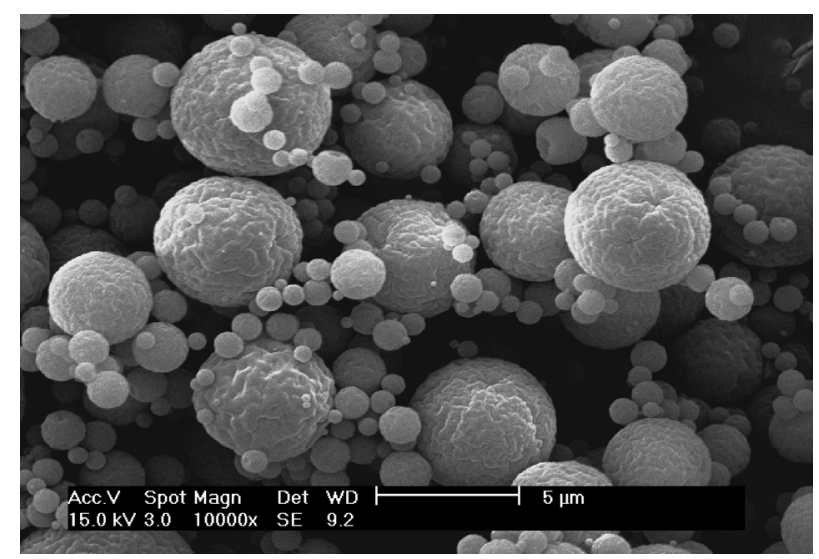

Fig. 3. Scanning Electron Microscopy of UEA-CS-NPs-MPS Prepared by a Spray Drying Method

mean diameter of $300 \mathrm{~nm}$ might be obtained from one or two CS nanoparticles (Fig. 3). Except for the small particles (less than $1 \mu \mathrm{m})$, mean diameter of the 'large' microparticles was concentrated around $3.5 \mu \mathrm{m}$. Overall, the largely differentiated size of NiMOS particles could be acceptable for further drug dosage formation (tablet or granular).

The spray-dried UEA-CS-NPs-MPS could be well re-dispersed in PBS. As to our early experiences, the hydrophilic characterization and enzymatic degradation property of bovine serum albumin microspheres changed little during the spray drying process. ${ }^{27,28)}$ The activity and sugar-specificity of UEA-CS-NPs-MPS might be well preserved, and were further evaluated in this work.

The Binding of BSM to UEA-CS-NPs-MPS The activity of lectin after coupling depends on its ability to interact freely with the substrate molecules. BSM is a glycoprotein whose carbohydrate part is formed by oligosaccharide chains composed of six different sugars. The interaction of UEACS-NPs-MPS with BSM could suggest the remained activity of the covalently coupled UEA.

It could be seen from Fig. 4 that the bound rates of BSM to UEA-CS-NPs-MPS were always higher than those observed in microparticles without lectin. As far as the amount of BSM concerned, the affinity of UEA-CS-NPs-MPS to BSM was superior to the non-conjugated particles. This phenomenon initially indicated that the activity of UEA could be well kept invariably after binding. As for the $100 \mu \mathrm{g}$ BSM added group, its binding capacity reached up to about $92 \%$ (Fig. 4). This high enough binding activity of UEA-CS-NPs- 


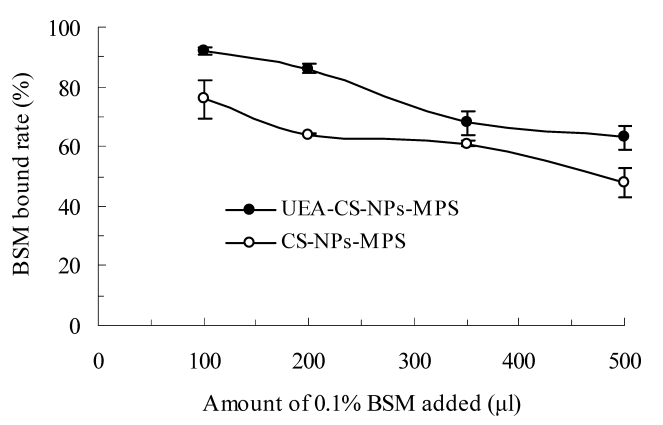

Fig. 4. The Effect of Different Amount of $0.1 \%$ Bovine Submaxillary Gland Mucin (w/v) on Its Coupling Capacity Bound to UEA-CS-NPs-MPS (20 mg Dispersed in $1 \mathrm{ml} \mathrm{PBS,} 15 \mu \mathrm{g} \mathrm{Lectin} / \mathrm{ml}$ ) and CS-NPs-MPS (20 mg Dispersed in $1 \mathrm{ml} \mathrm{PBS})(n=3)$

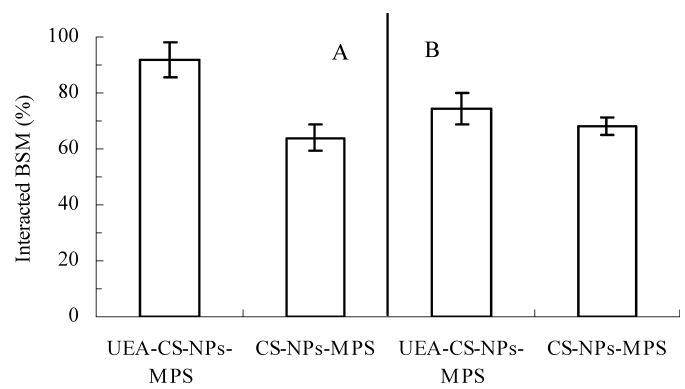

Fig. 5. BSM $(100 \mu \mathrm{l}, 1 \mathrm{mg} / \mathrm{ml})$ Binding to UEA-CS-NPs-MPS (20 mg Dispersed in $1 \mathrm{ml} \mathrm{PBS,} 15 \mu \mathrm{g}$ Lectin/ml) and CS-NPs-MPS (20 mg Dispersed in $1 \mathrm{ml}$ PBS) without (A) or with (B) $100 \mu \mathrm{mol}$ Fucose at Room Temperature $(n=3)$

MPS might promise further applications for the lectin mediated drug or vaccine delivery in specific regions of the GI tract.

As outlined in Fig. 4, a remarkable decrease in bound percent might be resulted from the increasing amount of BSM used. As for UEA-CS-NPs-MPS, the BSM bound rate decreased downward from $92 \%$ (100 $\mu \mathrm{l}$ BSM) to $62.8 \%$ (500 $\mu \mathrm{l}$ BSM). Such reduction in bound percent could also suggest the rational amount of BSM used for the next sugar competitive experiment.

The Competitive Inhibition of BSM Bound to UEA-CSNPs-MPS by Fucose The competitive studies with fucose as inhibitor would confirm the reported specific characteristics of UEA to $\alpha$-(L)-fucose. Figure 5 showed the amounts of BSM which interacted with the UEA-CS-NPs-MPS or CSNPs-MPS (used as control) in the absence and in the presence of fucose. The binding interactions between UEA-CSNPs-MPS and BSM decreased largely after addition of the specific sugar of fucose. At the sugar level of $100 \mu \mathrm{mol}$ fucose, the binding rate of BSM to UEA-CS-NPs-MPS was attenuated nearly by $20 \%$ (from 92 to $74.2 \%$ ) (Fig. 5). Wherever, the interaction was approximately similar to that obtained with control particles of CS-NPs-MPS. It seemed that these conjugates could have an important affinity for fucose in addition to the carbohydrate chain of BSM. These results clearly suggested the remained lectin activity after its covalent coupling to CS particles. Accordingly, the construction of UEA-CS-NPs-MPS could really keep the specific binding characteristics of UEA to fucose as for the well presented lectin residues.
It has been supposed that the lectin-anchored bioadhesive devices could be used to (i) increase residence time of the administered drug, (ii) improve bioavailability and efficiency of the therapies, and (iii) develop new therapeutic strategies upon site-specific delivery within the GI tract. For the above consideration, the design and preparation of lectin conjugates might be of interest for different target objectives within the mucosa such as epithelial cells, absorptive windows, cancerous cells and Peyer's patches or gut associated lymphoid tissue. $^{15)}$

\section{Conclusion}

This study has shown that CS-NPs are a feasible carrier for binding UEA lectin. The activity and sugar-specificity of UEA, demonstrated in this paper, was well preserved after covalent coupling and spray drying process. Therefore, the prepared UEA-CS-NPs-MPS could be used as a rational lectin-mediated mucosa drug delivery system. Its potential application for drug entrapment might be very interesting for efficiently improving the interactions between the pharmaceutical dosage form and different biological structures located on the mucosa. Meanwhile, the obtained results are quite encouraging in terms of further development of nanoparticles-in-microparticles for oral delivery of antigen to antigen presenting cells of the Peyer's patches.

Acknowledgements This work was supported by the NSFC (30500639) and the emphasized fundamental investigation project of Shanghai (08JC1406900).

\section{References}

1) O'Hagan D. T., Adv. Drug Deliv. Rev., 34, 305-320 (1998).

2) Chen H., Torchilin V., Langer R., Pharm. Res., 13, 1378-1383 (1996).

3) Gupta P. N., Mahor S., Rawat A., Khatri K., Goyal A., Vyas S. P., Int. J. Pharm., 318, 163-173 (2006).

4) Lis H., Sharon N., Annu. Rev. Biochem., 55, 35-67 (1986).

5) Kilpatrick D. C., Pusztai A., Grant G., Graham C., Ewen S. W., FEBS Lett., 185, 299-305 (1985).

6) Lehr C. M., Bouwstra J. A., Kok W., Noach A. B., de Boer A. G., Junginger H. E., Pharm. Res., 9, 547-553 (1992).

7) Vyas S. P., Singh A., Sihorkar V., Crit. Rev. Ther. Drug Carrier Syst., 18, 1-76 (2001)

8) Lehr C. M., J. Controlled Release, 65, 19-29 (2000).

9) Clark M. A., Hirst B. H., Jepson M. A., Adv. Drug Deliv. Rev., 43, $207-223$ (2000).

10) Irache J. M., Durrer C., Duchêne D., Ponchel G., Pharm. Res., 13, 1716 -1719 (1996).

11) Russell-Jones G. J., Veitch H., Arthur L., Int. J. Pharm., 190, 165174 (1999).

12) Gao X., Tao W., Lu W., Zhang Q., Zhang Y., Jiang X., Fu S., Biomaterials, 27, 3482-3490 (2006).

13) Lee A. L. Z., Wang Y., Ye W. H., Yoon H. S., Chan S. Y., Yang Y. Y., Biomaterials, 29, 1224-1232 (2008).

14) Ponchel G., Irache J., Adv. Drug Deliv. Rev., 34, 191-219 (1998).

15) Ezpeleta I., Arangoa M. A., Irache J. M., Stainmesse S., Chabenat C., Popineau Y., Orecchioni A. M., Int. J. Pharm., 191, 25-32 (1999).

16) Montisci M. J., Giovannuci G., Duchêne D., Ponchel G., Int. J. Pharm., 215, 153 - 161 (2001).

17) Illum L., Pharm. Res., 15, 1326-1331 (1998).

18) Bhavsar M. D., Tiwari S. B., Amiji M. M., J. Controlled Release, 110 , 422-430 (2006).

19) Bhavsar M. D., Amiji M. M., J. Controlled Release, 119, 339-348 (2007).

20) Bodmeier R., Chen H. G., Paeratakul O., Pharm. Res., 6, 413-417 (1989).

21) Fei Y. B., Li F. Q., Hu J. H., Liu J. Y., Zhao Y. Z., Pharm. Care Res., 8, 119-122 (2008).

22) Berthold A., Cremer K., Kreuter J., J. Controlled Release, 39, 17-25 
(1996).

23) Li F. Q., Su H., Wang J., Liu J. Y., Zhu Q. G., Fei Y. B., Pan Y. H., Hu J. H., Int. J. Pharm., 349, 274-282 (2008).

24) Irache J. M., Durrer C., Duchêne D., Ponchel G., Biomaterials, 15, 899-904 (1994)

25) Olde Damink L. H., Dijkstra P. J., van Luyn M. J., van Wachem P. B.,
Nieuwenhuis P., Feijen J., Biomaterials, 17, 679-684 (1996).

26) Monsan P., Puzo G., Mazarguil H., Biochimie, 57, 1281-1292 (1975)

27) Lu B., Li F. Q., Acta Pharm. Sin., 35, 535-538 (2000).

28) Li F. Q., Hu J. H., Lu B., Yao H., Zhang W. G., J. Microencapsul., 18, $825-829$ (2001). 